\title{
Disease Ontology Object
}

National Cancer Institute

\section{Source}

National Cancer Institute. Disease Ontology Object. NCI Thesaurus. Code C45370.

Disease objects specify a disease name and ID; disease objects also provide access to:

ontological relations to other diseases; clinical trial protocols treating the disease; and specific histologies associated with instances of the disease. 